Pfannkuch, Statistisches über den Einfluss des Puerperiums u. s. w. 367

Nacken um, und umschlingt ein Mal lose den Hals. Zur rechten Seite desselben zurïckgekehrt, krïmmt sie sich hackenförmig um denjenigen Theil des Nabelstranges, der die vorhin beschriebene Umbiegungsstelle bildet. Von dort nimmt sie ihren Weg über die Dorsalseite der Hand, legt sich zwischen Beugeseite des Vorder- and Oberarmes und tritt dann zum Nabel.

Die Nabelschnur hat in diesem Falle einen stärkeren Druck nur auf das linke Handgelenk ausgeübt, zeigt aber in ihrer ganz en Länge so bedentende Torsionen, besonders am Nabel, dass durch diese sicher der Tod erfolgte. Die am Halse befindliche eigenthümliche Verkettung der beiden dort zusammentretenden Schlingen möchte wohl bisher einzig in ihrer Art sein.

Fall IV (Figur 5 und 6).

Dieser Fall wurde im Jahre 1858 in der Entbindungsanstalt beobachtet.

Die am 14. October aufgenommene $\mathrm{H}$. wurde im VIII. Monate der Schwangerschaft geschätzt. Die Frucht befand sich in I. Schädellage, die Herztöne waren links hörbar.

Am 12. November erfolgte zwei Stunden nach Wehenbeginn die Geburt eines $2500 \mathrm{Gm}$. schweren, kurz vor der Geburt abgestorbenen Mädehens. Die Frucht war durch Strangulation des Halses zu Grunde gegangen. Die Nabelschnur umschlang nämlich drei Mal sehr fest den Hals des Kindes und war obendrein mit einem wahren Knoten, der seinen Sitz gerade in der Mitte des Halses in der zweiten Umschlingung hatte, versehen, Vom Halse verlief die Nabelschnur in gerader Richtung und starker Spannung zum Nabel, der etwas herausgezerrt war.

Der Hals war stark zusammengeschnürt. Die einzelnen Umschlingungen hatten tiefe Druckfurchen in Haut und Muskulatur hinterlassen.

\title{
Statistisches über den Einfluss des Puerperiums auf die Conceptionsfähigkeit.
}

\author{
Von \\ Dr. Wilhelm Pfannkuch
}

in. Cassel.

Die Conceptionsfähigkeit kann durch vorausgegangene Wochenbetten nach zwei Richtungen hin verändert, nämlich sowohl erhöht als vermindert werden, letzteres bis ziw völligen Vernichtung. 
Ueber den ersteren Einfluss sagt Olsha usen gelegentlich seines Vortrages "Ueber die blutige Erweiterung des Gebärmutterhalses" (S. 497): "Kein erfahrener Gynäkologe bestreitet heutzutage noch die Thatsache, dass die durch die erste Geburt gesetzte bleibende Erweiterung des Muttermundes auch für das ganze künftige Leben die Conception erleichtert. Gerade diejenigen Fälle sind hierfür am meisten beweisend, in welchen nach anfänglicher mehrjähriger Sterilität endlich, sei es mit oder ohne Kunsthülfe, Conception eingetreten war und nun schnell eine Conception der anderen folgt."

Andererseits lehrt die Erfahrung, dass zuweilen nach einmaliger rasch eingetretener Empfängniss eine ungewöhnlich lange Pause entsteht, oder dass eine zweite überhaupt nicht mehr vorkommt, für das übrige Leben also Unfruchtbarkeit die Folge ist. Sims giebt an, etwa ebenso oft erworbene wie angeborene Sterilität beobachtet zu haben.

Die Ursache dieser verminderten oder aufgehobenen Conceptionsfähigkeit liegt fast immer in pathologischen Veränderungen, welche in dem vorausgegangenen Puerperium sich entwickelt haben und die nun dem Eintritte neuer Empfängniss ein bald grösseres, bald geringeres physiologisches oder mechanisches Hinderniss bereiten.

Bei 240 Ehen mit mindestens je zwei Kindern (entnommen dem 76. Jahrgange des gothaischen genealogischen Taschenbuches auf das Jahr 1839) vergingen bis zu der Geburt des ersten Kindes im Mittel 24,5 Monate, und von da bis zur zweiten Entbindung 28,7 Monate. Eine Erleichterung der zweiten Conception durch die vorausgegangene Geburt geht aus dieser Gegenüberstellung nicht hervor. Natürlich muss man von den 28,7 Monaten einen Abzug machen für die Dauer des Puerperiums und etwaiger Lactation. Nehmen wir aber auch den günstigen Fall an, dass nämlich die Hälfte der Entbundenen sechs Monate gestillt habe, und machen wir bei ihnen für die Zeit bis zum Wiederbeginne der Ovulation dementsprechend einen Abzug von sechs Monaten, berechnen wir ferner dieselbe Zeit für die andere nicht stillende Hälfte auf volle zwei Monate, so würde der mittlere Abzug erst vier Monate d. i. gerade die Differenz der beiden oben gefundenen Zeiträume betragen. Es stellt sich also trotz dieses immerhin hoch berechneten Abzugs der zweite Zeitraum nicht kleiner heraus als der erste, und wir müssen deshalb schliessen, dass die Chancen für den Eiutritt neuer Conception durch die vorausgegangene Geburt im Allgemeinen nicht verbessert werden, sondern unverändert die gleichen bleiben. Es ist aber wenig wahrscheinlich, dass die Hälfte der Damen des gothaischen Kalenders sechs Monate gestillt hat. Mehrere haben bereits nach 10-12 Monaten und $28 \%$ nach höchstens 16 Monaten zum zweiten Male geboren. Etwa ebenso viele sind es, bei denen die zweite Geburt der ersten rascher folgte als die erste der Verheirathung.

Ziehen wir auch noch die späteren bis zur sechsten Entbindung in den Kreis unserer Betrachtung, so ergiebt sich folgende Tabelle: 
Es vergingen

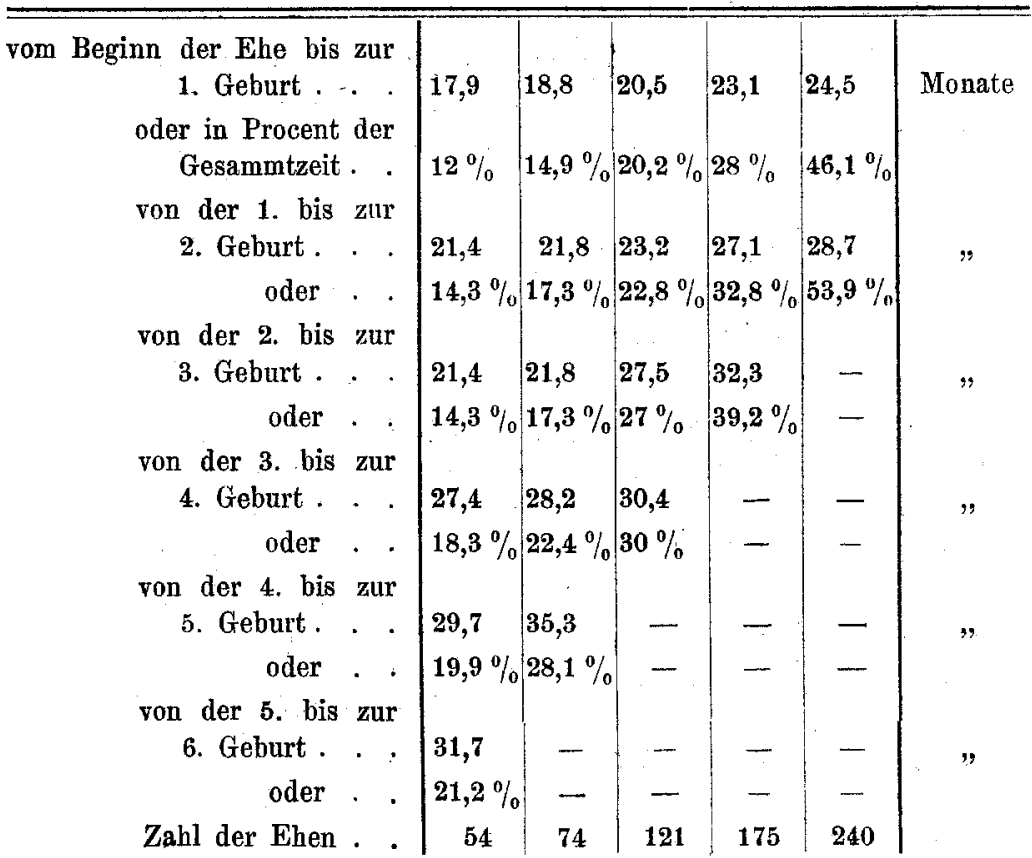

Man erkennt sofort, dass die Zahlen sowohl von links nach rechts als von oben nach unten grösser werden. Das Ansteigen in horizontaler Richtung bedarf kaum der Erklärung, denn es ist selbstverständlich, dass, je mehr Kinder einer Ehe entsprossen, desto kleiner die Zwischenräume zwischen den einzelnen Geburten sein müssen und umgekehrt. Das Ansteigen der Zahlen in verticaler Richtnng lehrt, dass mit der Zahl der Geburt auch die ihr vorausgehende Pause grösser wird. Zur richtigen Würdigung dieser Reihen ist jedoch zu bedenken, dass in dem gothaischen Kalender die Fehlund Todtgeburten nicht angegeben sind, zu welch' ersteren z. B. Multiparae viel mehr disponiren sollen, als Primiparae. (S chröder fand wenigstens auf 28 Multiparae nur 3 Primiparae. Lehrbuch 1872, 252.) Im Uebrigen erklärt sich diese Erscheinung dadurch, dass eine spätere Entbindung zur Erleichterung der Conception wohl nur ausnahmsweise noch etwas beizutragen vermag; mit jeder nenen Geburt muss vielmehr der empfängnisserschwerende' Einfluss immer mehr zur Geltung kommen, da mit der Zahl der Wochenbetten ja auch die Gelegenheiten zu pathologischen Veränderungen im Genitalsystem sich häufen.

Vergleichen wir nun diejenigen Ehen mit einander, in denen Sterilität entweder vor der ersten Entbindung vorhanden oder nach derselben entstanden war. Dabei verstehe ich unter Sterilität freie Zeiträume von mindestens fünf Jahren. 
370 Pfannkuch, Statistisches über den Einfluss des Puerperiums u. s. w.

Es vergingen vom Beginne der Ehe bis zur ersten Geburt im Durchschnitt

bei 23 Ehen 93,7 und von der 1. bis zur 2. Geburt 32,8 Monate,



Bei den 23 Ehen betrug der Zeitraum von der ersten bis zur zweiten Entbindung nur ein Mal wieder über fünf Jahre (82 Monate), und bei den 19 Fhen war die erste Entbindung dem Beginne der Ehe nur zwei Mal nach mehr als fünf Jahren (68 und 74 Monate) gefolgt.

Temporäre Sterilität scheint demnach durch die erste Entbindung etwa ebenso oft hervorgerufen als beseitigt zu werden, und zwar scheinen in beiden Fällen die durchschnittlichen Zwischenräume in nahezu gleichem Verhältnisse zu stehen, hier in dem von 1:3. Daher mag es kommen, dass bei der Vergleichung einer grösseren Zahl von Geburten der etwa vorhandene conceptionsbefördernde Einfluss der ersten Geburt nicht zum Ausdruck kommt.

Knabengeburten sind bekanntlich im Allgemeinen schwieriger als Mädchengeburten, und deshalb gefahrvoller für die Mütter. Eine dieser Thatsache entsprechende Einwirkung zeigt sich auch betreffs der Conceptionsfähigkeit. Nach 106 Knabengeburten vergingen nämlich durchschnittlich 30,2 Monate bis zur zweiten Entbindung, während dieselbe auf 134 Mädchengeburten schon nach 27,4 Monaten folgte.

Schliesslich erwähne ich noch, dass die Zahlen anch einen Einfluss des miitterlichen Alters auf die Conceptionsfähigkeit erkennen lassen. Es fand sich, dass 88 Franen, die im Laufe des ersten Jahres ihrer Ehe geboren, im Mittel 21,3 Jahre, dagegen 37 andere, die erst nach Ablauf von drei Jahren niederkamen, 20,7 Jahre alt waren. Deutlicher noch wird die Differenz in folgender Gruppirung. 127 Frauen, die bei ihrer Verheirathung bereits das 20. Lebensjahr übersehritten hatten, gebaren nach durchschnittlich 20,8 Monaten, 93 jüngere Frauen dagegen erst nach 26,5 Monaten.

Die Begründung dieser Erscheinung mag theils in mechanischen, theils in physiologischen Schwierigkeiten zu suchen sein, welche durch einen mehr infantilen Zustand der Genitalien der Conception einerund der Ernährung des Eies andererseits entgegengestellt werden. 